\title{
Infrared Light Induced Patterning of Proteins on PPNIPAM THERMORESPONSIVE ThIN FILMS: A "ProteIn LASER PRINTER"
}

\author{
Karl F. Böhringer ${ }^{1,2}$, Huseyin Bilge ${ }^{1}$, Xuanhong Cheng ${ }^{3}$, Buddy Ratner ${ }^{4}$, Shoji Takeuchi ${ }^{2}$, Hiroyuki Fujita ${ }^{2}$ \\ ${ }^{1}$ Department of Electrical Engineering, University of Washington, Seattle, WA, USA \\ ${ }^{2}$ Institute of Industrial Science, University of Tokyo, Komaba, Tokyo, JAPAN \\ ${ }^{3}$ Massachusetts General Hospital, Boston, MA, USA \\ ${ }^{4}$ University of Washington Engineered Biomaterials (UWEB), Seattle, WA, USA
}

\begin{abstract}
Controlled adsorption and patterning of proteins on a thin film of thermo-responsive plasma-deposited poly- $\mathrm{N}$-isopropylacrylamide occurs in response to heating with a computer-controlled infrared laser beam, with pattern resolution in the single micrometer range and adsorption times within a few seconds. Deposition of multiple proteins in the same substrate region has been accomplished. Successful streptavidin-biotin and antibody-antigen binding indicates that protein function stays intact after proteins adsorb to the ppNIPAM bio-polymer surface.
\end{abstract}

\section{INTRODUCTION}

Protein arrays play an important role in life science research, including proteomic profiling, protein detection assays, or drug testing $[1,2]$. Proteins in micro-arrays are typically deposited with techniques based on robotic contact printing [1], ink-jetting [3], photolithography [4], and dip-pen lithography $[5,6]$. High speed, high density, large array row and column count, large number of proteins, and easy reconfigurability are all important factors for the preparation of protein arrays. This paper presents an alternative method for protein patterning using laser-induced adsorption of proteins on a "smart polymer" thin film, leading to fast adsorption times and high resolution.

Prior work [7] has established that poly-N-isopropylacrylamide (pNIPAM) exhibits a distinct reversible phase transition at its lower critical solution temperature (LCST) of $31^{\circ} \mathrm{C}$ : below, it is hydrophilic and protein-repelling (non-fouling); above, it is hydrophobic and protein-adsorbing (bio-fouling). pNIPAM thermoresponsive thin films can be synthesized by vapor phase plasma deposition ("ppNIPAM") [8]. Thus, ppNIPAM can be conformally coated onto MEMS in a low-temperature process. Following this approach, selective patterning of proteins and cells has been accomplished on ppNIPAM coated microheaters [9-11]. By replacing the lithographically defined microheaters with a software-controlled laser beam, the work described in this paper simplifies the manufacture and use of the substrate surface by eliminating the need for electrical connections or for a microheater array addressing scheme; in addition, it improves the resolution of protein patterns and decreases the adsorption time, each by two orders of magnitude.

\section{EXPERIMENTAL SETUP}

Substrate fabrication: A 4" pyrex wafer is coated by evaporation with $100 \mathrm{~nm} \mathrm{Al}$, which forms an absorption layer for the IR laser. The $\mathrm{Al}$ is patterned into 22 different $1 \mathrm{~cm}^{2}$ regions; these regions consist of continuous $\mathrm{Al}$ or various dot patterns that reduce lateral heat conduction through the metal layer. This wafer is conformally coated with approximately $50 \mathrm{~nm}$ ppNIPAM [8]. 22 openings (footprint $1 \mathrm{~cm}^{2}$ ) are cut into a 4" diameter, 9mm thick polydimethylsiloxane (PDMS) mold that matches the $\mathrm{Al}$ patterns, and the mold is attached to the pyrex wafer such that it forms open chambers (volume 900 $\mu \mathrm{l}$ ) above each of the 22 patterns. An otherwise identical substrate without ppNIPAM coating is also fabricated for control experiments.

Laser setup: The wafer with PDMS chambers is placed onto an inverted microscope (Nikon Eclipse TE300) with motorized precision $x$-y-stage and IR laser (Sigma Koki optical tweezer, $\lambda=1064 \mathrm{~nm}$; Figs. 1,2 ). The laser shutter and stage are controlled via two USB interfaces from a laptop with custom software.

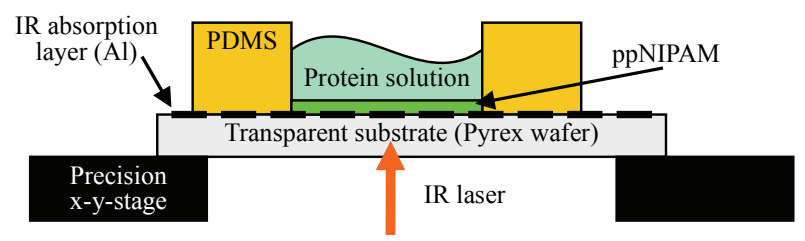

Fig. 1: Schematic cross-section of protein patterning chamber: pyrex wafer with Al layer for IR absorption, ppNIPAM thin film coating, PDMS chamber, and protein solution.

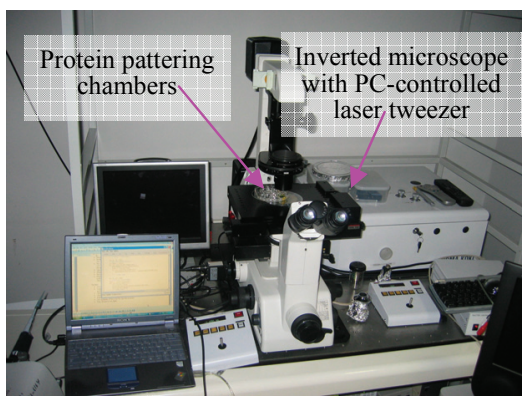

Fig. 2: Experimental setup with chambers on an inverted microscope with computer-controlled IR laser and motorized stage (positioning resolution $100 \mathrm{~nm})$. 
Protein preparation: Protein solution is manually dispensed by pipette into a PDMS chamber. The chamber is exposed to timed pulses of specific intensity from the IR laser while the microscope stage follows a software-defined path. Finally, the chamber is rinsed and protein adsorption is observed under a fluorescence microscope.

\section{TEMPERATURE CONTROL}

The phase transition of ppNIPAM occurs at $31^{\circ} \mathrm{C}$, conveniently located above room temperature but below a temperature at which proteins would denature. Thus it is important to control the dosage of the IR laser such that temperatures above $40^{\circ} \mathrm{C}$ are avoided. In addition, lateral heat conduction along the substrate surface should be minimized if high resolution protein patterns are to be achieved.

Accurate measurement and closed-loop control of the temperature at the location of the IR laser exposure is difficult because of the small spot size (a few micrometers in diameter), short exposure time, and aqueous environment. Instead, open-loop control is used for our experiments. In a simple initial calibration test, a small liquid crystal thermometer strip is placed at the bottom of a Petri dish with $5 \mathrm{~mm}$ water and heated with gradually increasing laser power until the temperature at the focal point exceeds $32^{\circ} \mathrm{C}$. After this approximate calibration, more accurate exposure tests with the IR laser can be performed, which will be described in Section 4.

Spatial temperature resolution is limited by the laser spot size (i.e., microscope optics and wavelength $\lambda=1064 \mathrm{~nm}$ ) and by the thermal conduction of the materials. To obtain an estimate for this conduction, we calculate the bulk or sheet resistances for the different layers from a heated spot with radius $r$ radially outward to a distance $d$.

Bulk thermal resistances (glass, water): $R=\frac{1}{2 \pi c}\left(\frac{1}{r}-\frac{1}{d}\right)$

Sheet thermal resistance (Al): $R=\frac{1}{2 \pi t c} \ln \frac{d}{r}$

The thermal conductivities for water, glass, and $\mathrm{Al}$ are, respectively, $c_{\text {water }} \approx 650 \mathrm{~W} / \mathrm{mK}, c_{\text {glass }} \approx 0.6 \mathrm{~W} / \mathrm{mK}, c_{\mathrm{Al}} \approx 236 \mathrm{~W} / \mathrm{mK}$, and $\mathrm{Al}$ thickness is $t=1000 \AA$. Numerical evaluation shows that the Al layer (even when not patterned) has the highest thermal resistance (e.g., for $r=1 \mu \mathrm{m}$ and $d=10 \mu \mathrm{m}$, $\left.R_{\text {water }}=6.7 \cdot 10^{3} \mathrm{~K} / \mathrm{W}, R_{\text {glass }}=1.6 \cdot 10^{6} \mathrm{~K} / \mathrm{W}, R_{\mathrm{Al}}=12 \cdot 10^{6} \mathrm{~K} / \mathrm{W}\right)$ and the path through water clearly dominates heat conduction.

\section{EXPERIMENTS AND OBSERVATIONS}

Three sets of successful experiments have been performed: (1) Deposition of fluorescently labeled bovine serum albumin (BSA), with the goal to directly observe protein adsorption in response to varying laser exposure times and intensities. (2) An identical experiment with an uncoated control substrate, to demonstrate that protein adsorption is indeed due to heated ppNIPAM. (3) Laser-induced adsorption of streptavidin and anti-BSA with subsequent binding assays of fluorescently labeled biotin and immunoglobulin $\mathrm{G}$ ( $\mathrm{IgG}$ ), respectively, to demonstrate that molecular recognition remains intact after adsorption to the heated ppNIPAM surface. In each case, exposure consists of executing a program where laser intensity varies from $100 \mathrm{~mW}$ to $800 \mathrm{~mW}$ optical power; exposure times from $1 \mathrm{~s}$ to $5 \mathrm{~min}$; and laser duty cycles from $25 \%$ to $100 \%$.

\subsection{Non-specific Protein Adsorption}

Experiment 4.1a: $50 \mu \mathrm{g}$ bovine serum albumin with fluorescein isothiocyanate conjugate (FITC BSA, Sigma A9771) dissolved in $500 \mu 110 \mathrm{mM}$ tris(hydroxymethyl)methylamine buffer (Tris Ultrapure, ICN Biochemicals) is pipetted into a chamber with continuous Al absorption layer and exposed. An exposure test with varying exposure times and laser pulse duty cycles is performed with a stationary laser (Fig. 3a), as well as several tests with the stage moving during exposure (Figs. 3b, c).

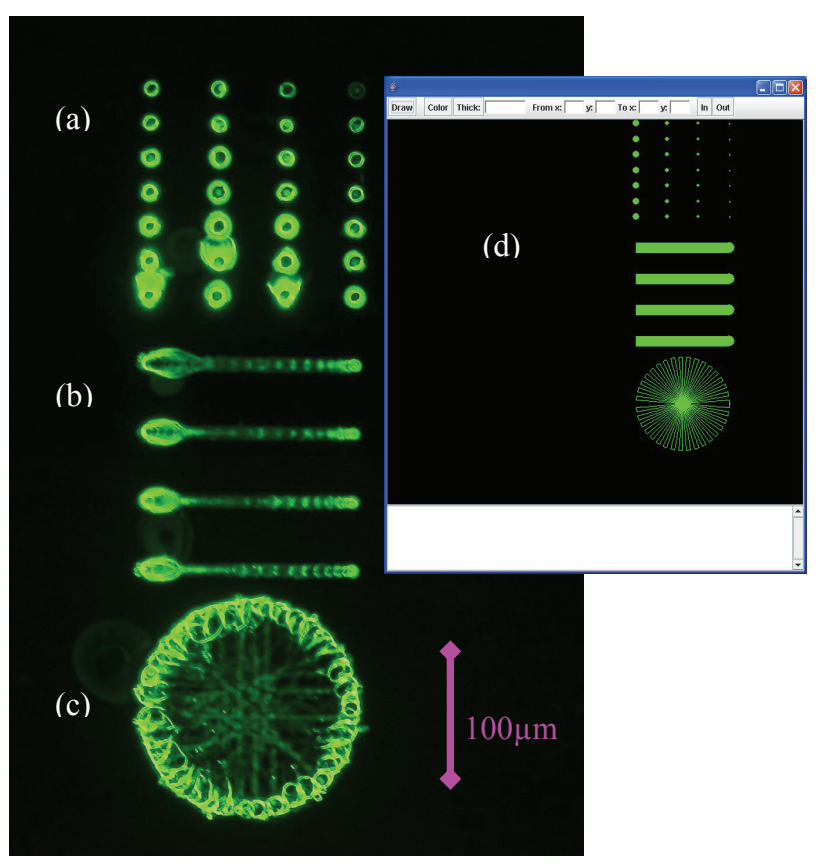

Fig. 3: FITC BSA adsorption with $600 \mathrm{~mW}$ optical power. (a) Rows: exposure times 1, 2, 5, 10, 20, 30, $60 \mathrm{sec}$ (top to bottom). Columns: laser duty cycle 100, 75, 50, $25 \%$ (left to right). (b) Linear motion with varying stage speed during

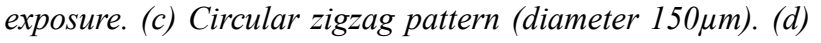
Graphical user interface of laser controller software.

Observations: Proteins adsorb to heated ppNIPAM surface, and adsorption can be detected via fluorescence microscopy after one second at $600 \mathrm{~mW}$ optical power, pulsed at a duty cycle of $25 \%$. Higher exposures have large protein build-up in an extended region around the laser spot (“over-exposure"). 
Note: proteins tend to adsorb in small ring shapes rather than points, likely because the focus of the IR laser is slightly different from the optical microscope focus.

Experiment 4.1b: The same BSA solution is used in an exposure test with varying exposure times, optical power, and magnification settings (Fig. 4).

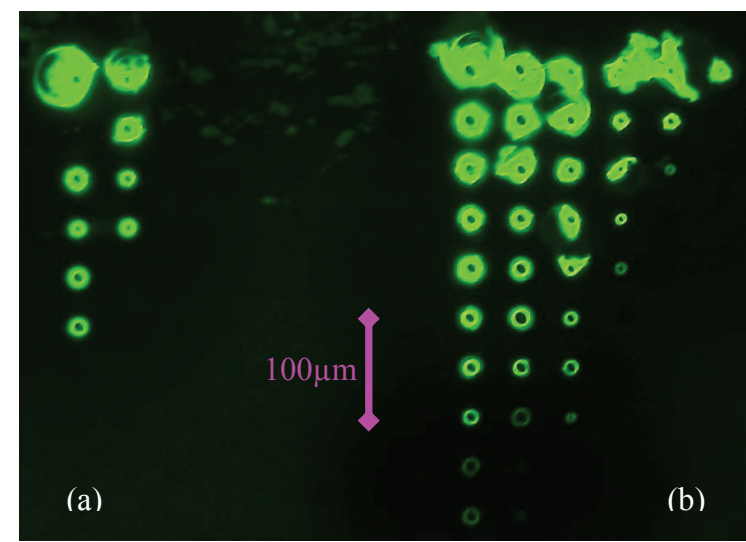

Fig. 4: Exposure tests with FITC BSA and (a) 40x lens, (b) $10 x$ lens. Rows: exposure times 1, 2, 3, 5, 10, 20, 30, 60, 120 , $300 \mathrm{sec}$ (bottom to top). Columns: optical power 100, 200, 300, 400, 500, $600 \mathrm{~mW}$ (right to left). Spacing between exposures is $50 \mu \mathrm{m}$.

Observations: Fastest adsorption is for $1 \mathrm{sec}$ at $600 \mathrm{~mW}$ (bottom left spot of 10x exposure), but even much lower intensities $(100 \mathrm{~mW})$ show deposition within minutes. Higher exposures again show "over-exposure". Optical loss in the laser beam is higher with the $40 \mathrm{x}$ objective lens.

\subsection{Control Experiments}

Experiment 4.2a: An experiment identical to 4.1 is performed with the control wafer that lacks the ppNIPAM coating. No laser-induced protein adsorption is observed.

Experiment 4.2b: An experiment analogous to 4.1 is performed on a substrate region with a discontinuous Al dot pattern. For each exposure, the laser is visually aligned with the center of an $\mathrm{Al}$ dot. The resulting protein patterns are virtually identical to experiment 4.1 , consistent with the calculations in Section 3, which predict similar heat distributions for patterned and unpatterned $\mathrm{Al}$ layer.

\subsection{Protein Adsorption and Specific Binding}

Experiment 4.3a: $500 \mu \mathrm{g}$ streptavidin dissolved in $500 \mu \mathrm{l}$ Tris is pipetted into a chamber and exposed; then the chamber is thoroughly rinsed with Tris and $200 \mu \mathrm{l}$ of $8 \mu \mathrm{M}$ fluorescently labeled biotin (biotin-4-fluorescein, Molecular Probes B-10570) is added for $1 \mathrm{~min}$ before additional rinsing.

Observations: Streptavidin adsorbs to the heated ppNIPAM surface, and its biotin-binding capability remains intact (Fig. 5). Adsorption was highest for the smallest ring (Fig. 5 left), where IR dosage is highest. No significant adsorption is visible for the large ring (right), which received the lowest IR dosage per area.

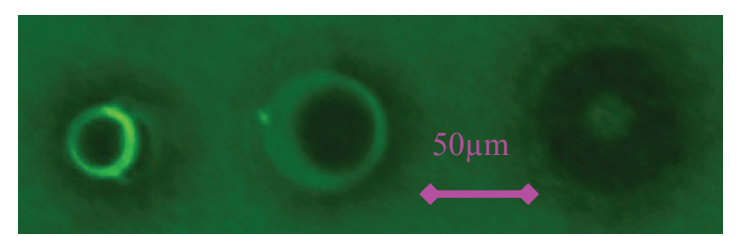

Fig. 5: Small ring patterns of adsorbed streptavidin with subsequent binding to fluorescein-labeled biotin. Rings were exposed with $500 \mathrm{~mW}$ optical power at a constant angular velocity of $45 \% \mathrm{sec}$ for $80 \mathrm{sec}$.

Note: ppNIPAM areas neighboring heated regions and areas exposed to low heating appear darker than the background (Fig. 5). This effect may be due to convection in the protein solution as the ppNIPAM surface approaches the LCST.

Experiment 4.3b: $900 \mu \mathrm{l}$ anti-BSA (lyophilized antiserum, Sigma B7276, produced in rabbit) with concentration $0.1 \mathrm{mg} / \mathrm{ml}$ in deionized water is pipetted into a chamber and exposed. Then the chamber is rinsed and blocked with $1 \%$ BSA in Dulbecco's phosphate-buffered saline (D-PBS) for several minutes. $900 \mu 1$ secondary antibody (donkey anti-rabbit IgG $(\mathrm{H}+\mathrm{L})$ fluorescein, Jackson Immunoresearch 711-095-152) diluted 1:35 in 1\% BSA D-PBS is pipetted into the chamber and reacted at room temperature for $30 \mathrm{~min}$ and subsequently rinsed with $1 \%$ BSA D-PBS.

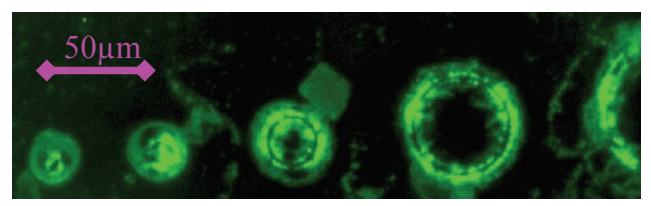

Fig. 6: Anti-BSA patterned onto ppNIPAM in laser-defined patterns. Fluorescently labeled secondary antibody $\operatorname{Ig} G$ binds to anti-BSA after patterning.

Observations: Anti-BSA adsorbs to the heated ppNIPAM surface, and the fluorescently labeled secondary antibody selectively binds to it, indicating that the protein function remains intact after adsorption (Fig. 6).

\subsection{Additional Observations}

Permanent protein adsorption: Even though the phase transition of pNIPAM is reversible, the protein binding appears to be irreversible. Without this favorable effect, which has been observed previously [9-11], proteins would desorb from the substrate after it cools down to room temperature.

"Protein color printer": The non-specific binding property of ppNIPAM allows multiple exposure-adsorption-rinse cycles to be performed in sequence. BSA, anti-BSA, and streptavidin have been patterned subsequently in the same substrate region. The previously deposited proteins are not significantly affected by later deposition cycles, as long as the exposure patterns do not overlap. 


\section{CONCLUSIONS AND FUTURE WORK}

IR laser induced, non-specific, irreversible adsorption of different types of proteins was accomplished on thermoresponsive ppNIPAM (with similarity to color laser printing). Functionality of the adsorbed protein remained intact. Specificity of adsorption can be achieved by a two-step binding protocol (e.g., streptavidin-biotin, or antibody-antigen pairs). Adsorption occurs within seconds of laser exposure. Patterns with features in the single micrometer range have been accomplished; further improvements in resolution appear possible.

The protein patterning approach presented here causes proteins from a solution to adsorb to a surface. Unlike inkjet or microcontact printing methods, no printhead needs to be in the immediate vicinity of the patterned region. Thus, this approach is related to freeform prototyping, and it could be used to pattern proteins inside channels or onto three-dimensional structures.

While protein adsorption on ppNIPAM is irreversible even after laser heating subsides, other studies with ppNIPAM have shown that cells immobilized via proteins on a ppNIPAM substrate detach when the temperature drops below the LCST $[10,11]$. This effect, which has been attributed to the larger mechanical stress exerted by the cells on the adsorption layer, could be used for controlled capture and release of cells in a biological assay.

Future applications of this technology may include low-cost diagnostic devices with protein arrays; precisely located capture of specific cells linked to adsorbed proteins; self-aligning adsorption of analytes in surface plasmon resonance (SPR) sensors [12]; and patterning of bio-catalysts for controlled three-dimensional synthesis of heterogeneous materials.

\section{ACKNOWLDGEMENTS}

The authors are grateful for the help by Winston Ciridon with the ppNIPAM preparation, Hiroaki Kitagawa with the laser system, Momoko Kumemura with PDMS processing, Cagatay Tahan and Agnes Tixier-Mita with chemical preparations and fluorescence microscopy, and Ji Hao Hoo, Tsung-Hao Su, and Jim Qi Zheng with Java programming of the graphical user interface for the laser controller.

This work received support from by the NIH Center of Excellence in Genomic Science and Technology grant 1-P50-HG002360-01. Karl Böhringer acknowledges a fellowship from the Japan Society for the Promotion of Science and the generous hospitality during his stay at the laboratories of Professors Hiroyuki Fujita, Shoji Takeuchi (University of Tokyo) and Osamu Tabata (University of Kyoto).

\section{REFERENCES}

[1] G. MacBeath and S. L. Schreiber, "Printing Proteins as Microarrays for High-Throughput Function Determination," Science, vol. 289, pp. 1760-1763, 2004.
[2] H. Zhu, M. Bilgin, R. Bangham, D. Hall, A. Casamayor, P. Bertone, N. Lan, R. Jansen, S. Bidlingmaier, T. Houfek, T. Mitchell, P. Miller, R. A. Dean, M. Gerstein, and M. Snyder, "Global Analysis of Protein Activities Using Proteome Chips," Science, vol. 293, pp. 2101-2105, 2001.

[3] J. F. Mooney, A. J. Hunt, J. R. McIntosh, C. A. Liberko, D. M. Walba, and C. T. Rogers, "Patterning of Functional Antibodies and other Proteins by Photolithography of Silane Monolayers," Proceedings of the National Academy of Science, vol. 93, pp. 12287-12291, 1996.

[4] G. Torsten and S. G. Juan, "DNA-printing: Utilization of a Standard Inkjet Printer for the Transfer of Nucleic Acids to Solid Supports," J. Biochem. Biophys. Methods, vol. 42, pp. 105-110, 2000.

[5] R. D. Piner, J. Zhu, F. Xu, S. H. Hong, and C. A. Mirkin, "'Dip-pen" Nanolithography," Science, vol. 283, pp. $661,1999$.

[6] J.-M. Nam, S. W. Han, K.-B. Lee, X. Liu, M. A. Ratner, and C. A. Mirkin, "Bioactive Protein Nanoarrays on Nickel Oxide Surfaces Formed by Dip-Pen Nanolithography," Angewandte Chemie International Edition, vol. 43, pp. 1246-1249, 2004.

[7] M. Heskins and J. E. Guillet, "Solution properties of poly(N-isopropylacrylamide)," Journal of Macromolecular Science, Part A-Pure and Applied Chemistry, vol. A2, pp. 1441, 1968.

[8] Y. V. Pan, R. A. Wesley, R. Luginbuhl, D. D. Denton, and B. D. Ratner, "Plasma polymerized

N-Isopropylacrylamide: Synthesis and characterization of a smart thermally responsive coating," Biomacromolecules, vol. 2, pp. 32-36, 2001.

[9] Y. Wang, X. Cheng, Y. Hanein, B. Ratner, and K. F. Böhringer, "Protein Patterning with Programmable Surface Chemistry Chips," presented at The Sixth International Conference on Miniaturized Chemical and Biochemical Analysis Systems (Micro TAS), Nara, Japan, 2002.

[10] Y. Wang, X. Cheng, Y. Hanein, A. Shastry, D. D. Denton, B. D. Ratner, and K. F. Böhringer, "Selective Attachment of Multiple Cell Types on Thermally Responsive Polymer," presented at The 12th International Conference on Solid-State Sensors and Actuators (Transducers'03), Boston, MA, 2003.

[11] X. Cheng, Y. Wang, Y. Hanein, K. F. Böhringer, and B. D. Ratner, "Novel Cell Patterning Using Microheater Controlled Thermoresponsive Plasma Films," Journal of Biomedical Materials Research, 2004.

[12] C. Boozer, Q. Yua, S. Chen, C.-Y. Lee, J. Homola, S. S. Yee, and S. Jiang, "Surface functionalization for self-referencing surface plasmon resonance (SPR) biosensors by multi-step self-assembly," Sensors and Actuators B: Chemical, vol. 90, pp. 22-30, 2003. 